Article

\title{
Quantifying Power System Operational Reliability
}

\author{
Nahakul Nepal and Rajesh Karki * \\ Power Systems Research Group, Department of Electrical and Computer Engineering, \\ University of Saskatchewan, Saskatoon, SK S7N 5A2, Canada \\ * Correspondence: rajesh.karki@usask.ca
}

Received: 28 April 2019; Accepted: 8 July 2019; Published: 10 July 2019

\begin{abstract}
Rising uncertainty in power systems due to ongoing system and operational changes has led to increasing risks in operating a system in a reliable and economic manner. There are growing reliability concerns with the widely used methods, such as the N-1 criterion, to determine operating reserve requirement during unit commitment, and the economic load dispatch method to allocate regulating margin to respond to disturbances. These deterministic methods do not consider the uncertainty or stochastic nature of power systems and are often inadequate to maintain the required operating reliability of modern power systems. This paper introduces a reliability index, designated as the committed generators' response risk (CGRR) that can be used to maintain a specified level of operating reliability. A probabilistic method to evaluate the CGRR is presented and validated using a Monte Carlo simulation technique. An application of the new index and the methodology is illustrated using the IEEE Reliability Test System. The CGRR provides a comprehensive operating risk measurement of the committed generation until further assistance is available to support the system, and therefore helps operators in decision making for unit commitment and dispatch of the generating units to meet the projected load in the short future time.
\end{abstract}

Keywords: power system reliability; operating risk; regulating margin; unit commitment; load dispatch; Monte Carlo simulation

\section{Introduction}

The ability of a power system to maintain the continuity of power supply to its customers mainly depends on the implementation of operating decisions, provided that the system is adequately planned. Determining the optimum number, type, and capacity of generating units that must be operated and deciding how these units should be dispatched to meet the projected load are two key questions that must be prudently answered by the system operator before making operating decisions on these two tasks, commonly known as unit commitment and load dispatch, respectively. The power generated by the operating or "committed" units at a given time must be equal to the load at the same instant. The balance between supply and demand of power can be disturbed by random and sudden changes in load or generation due to incidents, such as an outage of a committed unit. The ability of the system to respond quickly to these disturbances to regain the balance and maintain the continuity of power supply will depend on the load dispatch decision taken prior to the disturbances, which in turn will depend on the unit commitment decision that was taken prior to the load dispatch. The continuity of the power supply or the reliability of the operating system condition will therefore depend on both the unit commitment and load dispatch decisions. Both of these tasks should therefore be considered in the operating system reliability evaluation.

Many power system operators have conventionally used a deterministic criterion, such as the $\mathrm{N}-1$ [1], to assess the operating reserve required during unit commitment. Many utilities operating within the North American Reliability Corporation (NERC) guidelines use this criterion to determine 
their minimum operating reserve requirements. As deterministic methods do not consider the stochastic nature of the unit failures and load variations, these methods can result in high operational cost or poor operating reliability due to over scheduling or under scheduling of the generators, respectively. The committed generating units must be able to provide sufficient response capacity in a short time of several minutes, referred to as the response time, to regain power balance and provide necessary frequency control [2] after a disturbance occurs. This response capacity, or regulating margin (RM), that can be obtained within the response time is generally estimated deterministically based on operator experience. With the use of these deterministic methods, the inconsistency in system operating risk will further increase as uncertainty in power systems increases due to ongoing system changes, such as the rapid growth of wind power. Most system operators use the economic load dispatch (ELD) method to make decisions on dispatching the committed units to serve the load. This deterministic method minimizes the operating cost. As reliability and economics often compete with each other, the dispatch decisions based on this method can result in unacceptable reliability.

The above concerns have led to research and development of probabilistic methods that can incorporate the uncertainties in system operation and provide proper evaluation of system operating risks. The Pennsylvania-New Jersey-Maryland (PJM) method [3] provided pioneer work in applying probabilistic methods in operating reserve evaluation. A probability distribution of committed generation is created in this method as a function of the lead time. It is the exposure time of the operating components to random failures in a given operating condition, within which time additional capacity assistance will not be available. The unit commitment risk (UCR) index quantifies the operating risk associated with the unit commitment decision, which is the probability of the committed generation just meeting or failing to meet the load requirement [1]. The PJM method was extended to incorporate rapid start units in [4] and import/export constraints in [5]. The technique was further developed in [6] for spinning reserve assessment in a composite power system evaluation. A hybrid method proposed in [7] presents a probabilistic framework to evaluate the probabilities of finding the system in "healthy", "marginal", and "at risk" operating states that are classified using the N-1 deterministic criterion. This technique is utilized to assess spinning reserve requirements in wind connected power systems in [8]. The hybrid method is further modified in [9] for spinning reserve evaluation in a deregulated market.

Probabilistic methods have also been developed to assess the risk associated with the allocation of the spinning reserve to the committed units with varying ramp rates and capacity constraints. The probability of failing to generate sufficient response capacity within the specified margin time to satisfy the load following a disturbance is termed as the response risk (RR) [10]. This index is utilized in [11] for advanced dispatch of spinning reserve. A hybrid method is presented in [12] for optimal distribution of spinning reserve in a generating system.

Unit commitment and load dispatch are treated as two distinct operating tasks with separate risk criteria for decision making in published probabilistic methods for operational reliability. A UCR criterion-based unit commitment may restrict a desired dispatch with non-deployable spinning reserve due to ramp rate constraints of the generating units that are not considered in UCR evaluation. As the probability of power outage following a disturbance depends on risks associated with both of these tasks, a comprehensive risk index is necessary, and introducing such an index and developing a methodology for its application in operating decisions are the major contributions of this paper. A comprehensive response risk of committed generating units for a specified lead time is introduced in this paper and is designated as the committed generators' response risk (CGRR). An analytical method to evaluate the CGRR is presented and validated using a Monte Carlo simulation technique. An application of the new index and methodology is illustrated using the IEEE Reliability Test System (IEEE-RTS) [13], and the impact on operating reliability of important operating variables, such as number and size of committed units, unit ramp rates, regulating margin, and reserve distribution, are analyzed. The CGRR quantifies the comprehensive operating risk of the scheduled generation, considering all possible disturbances until further assistance is available to the system, and therefore 
provides a single reliability index to help operators in both the unit commitment and dispatch decisions of the generating units to meet the projected load in the short future time.

\section{Proposed Operating Reliability Assessment Model}

A power system is operated to respond to the stochastic nature of system component failures and/or load variations to continuously match the supply of power to the system demand in real time. Large variations that pose a risk of load curtailment are termed as disturbances in this paper. Forced outages of committed units can lead to load curtailment situations, and are therefore considered as the major disturbance events in this paper. The operational reliability methodology presented in this paper is scoped to the bulk system operator's jurisdiction where utilities are required to meet the N-1 transmission planning criteria, such that a single order line contingency does not curtail the ability of the system to serve the bulk-load points. The probability of multiple transmission contingencies in a short operational exposure time is very small. The transmission lines are therefore not considered in this study. Similarly, the load variations are neglected in this study since the load uncertainty in the short time from the known initial condition is insignificant. For any kind of disturbance, such as a generating unit outage, the healthy operating units must respond and fulfill the lost power within a specified response time to avoid load curtailment situations. The probability of such load curtailment situation depends on the probability and magnitude of power disturbances, the amount of operating reserve in the system, ramp rates and responding capacity of the units carrying the reserve, and the lead time after which the system will receive further capacity assistance. All of these parameters are therefore considered in developing an operating system reliability model to evaluate the comprehensive operating risk, designated as the CGRR in this paper.

Figure 1 portrays the underlying concepts in the evaluation of the CGRR index. An operating condition has $m$ number of generating units committed for a lead time $T_{L}$. It is shown in Figure 1 that $D_{i}$ is the disturbance at time $T_{i}$ associated with the $i^{\text {th }}$ event out of the total $2^{\mathrm{m}}$ contingencies, and $T_{R}$ is the response time within which the healthy generating units must respond with sufficient capacity to avoid a load curtailment situation.

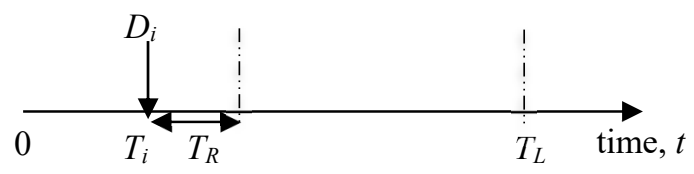

Figure 1. Conceptual illustration of CGRR evaluation of an operating condition.

The outage probability of a single committed unit is given by (1), and is known as the outage replacement rate (ORR) [1]:

$$
O R R_{i}=P\left(D_{i, i \in F}\right)=1-e^{-\lambda_{i} T_{L}}
$$

where, $i \in F$ includes all first order contingencies of unit outages, and $\lambda_{i}$ is the failure rate of unit $i$.

A discrete cumulative distribution function $F(Z)=P\left(Z_{k=1 \text { to } s}\right)$ of $s$ capacity outage states within the lead time $T_{L}$ can be obtained using (2) to recursively calculate the cumulative probability of $Z_{k} \mathrm{MW}$ outage in committed capacity [1].

$$
P\left(Z_{k}\right)=\left(1-O R R_{j}\right) * P^{\prime}\left(Z_{k}\right)+O R R_{j} * P^{\prime}\left(Z_{k}-C_{j}\right)
$$

where $C_{j}$ and $\mathrm{ORR}_{j}$ are the capacity and ORR for $T_{L}$ lead time respectively of unit $j$ added recursively to modify $F(Z) ; P^{\prime}\left(Z_{k}\right)$ and $P\left(Z_{k}\right)$ are the cumulative probabilities of $Z_{k}$ capacity outage before and after adding the $j^{\text {th }}$ unit. Equation (2) is initialized by setting $P^{\prime}\left(Z_{k}\right)=1$ for $Z_{k} \leq 0$ and $P^{\prime}\left(Z_{k}\right)=0$ otherwise [1]. 
A capacity outage $Z_{k}$ due a major disturbance $D_{i}$ results in a reduction in operating capacity $X_{k}$ given by (3), where $m$ is the number of committed units, $N$ is the total number of healthy operating units after the disturbance and $c_{L j}$ is the loading capacity of unit $j$ prior to the disturbance.

$$
X_{k}=\sum_{j=1}^{m-N} c_{L j}
$$

The probability of capacity $X_{k}$ reduction can then be obtained from the discrete probability density function $f(Z)=p\left(Z_{k=1 \text { to s }}\right)$, where $p\left(X_{k}\right)=P\left(Z_{k}\right)-P\left(Z_{k-1}\right)$. When a disturbance occurs, resulting in capacity drop $X_{k}$, the healthy units carrying spinning reserve will respond to fulfill the capacity mismatch. The response capacity, or the available regulating margin (RM) of the operating system in the response time $T_{R}$ following the $k^{\text {th }}$ disturbance is given by (4).

$$
R M_{k}=\sum_{j=1}^{N} R M_{j}
$$

where $N$ is the total number of healthy operating units, and $\mathrm{RM}_{j}$ is the response capacity of unit $j$ in the response time $T_{R}$ or the regulating margin of the unit given by (5), where $\gamma_{j}$ and $M C R_{j}$ are the ramp rate and maximum continuous rating of unit $j$.

$$
R M_{j}=\operatorname{MIN}\left(\gamma_{j} \times T_{R}, M C R_{j}-c_{L j}\right)
$$

The response risk associated with capacity drop $X_{k}$ is the conditional probability given by (6).

$$
\begin{aligned}
R R \mid Z_{k} & =0 \text { if } R M_{k}>X_{k} \\
& =1 \text { otherwise }
\end{aligned}
$$

The CGRR can then be evaluated using a conditional probability approach as shown in (7) by aggregating the conditional response risk obtained in (6) weighted by the probability $p\left(Z_{k}\right)$ for all $s$ outage states in the discrete distribution $f(Z)$.

$$
C G R R=\sum_{k=1}^{s} p\left(Z_{k}\right) \times R R \mid Z_{k}
$$

\section{Illustration of the Proposed Method}

The proposed methodology is illustrated in this section by application to a small generating system with four units committed for a lead time of $1 \mathrm{~h}$. The unit capacity rating $M C R$, loading capacity $c_{L}$, failure rate $\lambda$, and ramp rate $\gamma$ are shown in Table 1 . The regulating margin (RM) shown in the last column is calculated using (4) considering a response time $T_{R}$ of $10 \mathrm{~min}$. The total regulating margin is $30 \mathrm{MW}$. This will however be reduced by disturbances caused by unit outages. The unit commitment shown in Table 1 meets the N-1 criterion, as the spinning reserve of $36 \mathrm{MW}$ is equal to the MCR of the largest unit.

Table 1. Example operating system consisting of four generating units.

\begin{tabular}{ccccccc}
\hline $\begin{array}{c}\text { Units } \\
\boldsymbol{j}\end{array}$ & $\begin{array}{c}\text { MCR } \\
\mathbf{( M W )}\end{array}$ & $\begin{array}{c}\text { Failure Rate } \\
\boldsymbol{\lambda}(\mathbf{f} / \mathbf{y r})\end{array}$ & $\begin{array}{c}\text { Ramp } \\
\text { Rate } \boldsymbol{\gamma}(\mathbf{M W} / \mathbf{m})\end{array}$ & $\begin{array}{c}\text { Loading } C L \\
\mathbf{( M W )}\end{array}$ & $\begin{array}{c}\text { Spinning Reserve } \\
(\mathbf{M W})\end{array}$ & $\begin{array}{c}\text { RM } \\
(\mathbf{M W})\end{array}$ \\
\hline 1 & 20 & 1 & 2 & 7 & 13 & 13 \\
2 & 36 & 1 & 1 & 20 & 16 & 10 \\
3 & 11 & 1 & 1 & 4 & 7 & 7 \\
4 & 33 & 1 & 1 & 33 & 0 & 0 \\
Total & 100 & & & 64 & 36 & 30 \\
\hline
\end{tabular}


There exist $2^{4}=16$ contingences of unit outages, as shown in Table 2, considering 2-state generation models. The ORR for each unit in Table 1 is calculated using (1) for a lead time of $1 \mathrm{~h}$ and used in (2) to obtain the discrete probability distribution of capacity outages shown in the second and fourth columns of Table 2 . The reduction in operating capacity $X_{k}$ obtained from (3) are shown in the third column, and system response capacities or available regulating margins $\mathrm{RM}_{k}$ are obtained from (4) on the fifth column of Table 2 . The conditional response risk for each capacity outage condition shown in the sixth column are obtained using (6), and the CGRR shown is calculated using (7).

The obtained CGRR value of 0.00022837 is the total probability that generating units committed for the given operating decision will not be able to respond with adequate generation capacity to satisfy the system load within acceptable response time following major disturbances that can occur, considering all contingencies of generation unit outages. It should be noted that this risk can be calculated prior to making the operating decision on unit commitment and load dispatch, and the risk can be varied by an alternate decision to meet an acceptable criterion.

Table 2. Illustration of CGRR evaluation of the example system.

\begin{tabular}{|c|c|c|c|c|c|c|}
\hline State $k$ & Outage MW $Z_{k}$ & Reduction MW $X_{k}$ & Probability $p\left(Z_{k}\right)$ & RM MW & $R R \mid Z_{k}$ & $p\left(Z_{k}\right) \times R R \mid Z_{k}$ \\
\hline 1 & 0 & 0 & 0.9993152 & 30 & 0 & 0 \\
\hline 2 & 11 & 4 & 0.0001141 & 23 & 0 & 0 \\
\hline 3 & 20 & 7 & 0.0001141 & 17 & 0 & 0 \\
\hline 4 & 31 & 11 & $1.30 \times 10^{-8}$ & 10 & 1 & $1.30 \times 10^{-8}$ \\
\hline 5 & 33 & 33 & 0.0002282 & 30 & 1 & 0.0002282 \\
\hline 6 & 36 & 20 & 0.0002282 & 20 & 0 & 0 \\
\hline 7 & 44 & 43 & $2.61 \times 10^{-8}$ & 33 & 1 & $2.61 \times 10^{-8}$ \\
\hline 8 & 47 & 24 & $2.61 \times 10^{-8}$ & 13 & 1 & $2.61 \times 10^{-8}$ \\
\hline 9 & 53 & 40 & $2.61 \times 10^{-8}$ & 17 & 1 & $2.61 \times 10^{-8}$ \\
\hline 10 & 56 & 27 & $2.61 \times 10^{-8}$ & 7 & 1 & $2.61 \times 10^{-8}$ \\
\hline 11 & 64 & 44 & $2.97 \times 10^{-12}$ & 10 & 1 & $2.97 \times 10^{-12}$ \\
\hline 12 & 67 & 31 & $2.97 \times 10^{-12}$ & 0 & 1 & $2.97 \times 10^{-12}$ \\
\hline 13 & 69 & 53 & $5.21 \times 10^{-8}$ & 20 & 1 & $5.21 \times 10^{-8}$ \\
\hline 14 & 80 & 57 & $5.95 \times 10^{-12}$ & 13 & 1 & $5.95 \times 10^{-12}$ \\
\hline 15 & 89 & 60 & $5.95 \times 10^{-12}$ & 7 & 1 & $5.95 \times 10^{-12}$ \\
\hline 16 & 100 & 64 & $6.79 \times 10^{-16}$ & 0 & 1 & $6.79 \times 10^{-16}$ \\
\hline & & & & & \multicolumn{2}{|c|}{ CGRR $=0.00022837$} \\
\hline
\end{tabular}

\section{Simplification of the Proposed Method}

The illustrative example in Table 2 considers only four committed units in the operating condition. The number of units can be quite large in a practical system operation which will result in a large number of contingency states and significantly increase computation burden. The computation effort can be reduced by neglecting higher order contingencies that have a very low probability of occurrence. It is noted in Table 2 that states 11, 12,14, 15, and 16 are associated with third and fourth order contingencies involving outages of three and four units, respectively, within the exposure time. It can be observed that the CGRR will remain the same, within eight decimal places, if these higher order states are not considered in the evaluation in Table 2.

A study was carried out considering different loading conditions of the IEEE-RTS to determine an appropriate number of contingencies to be included in the CGRR evaluation in order to reduce computation effort while achieving reasonable accuracy. Table 3 shows nine operating schedules to meet the load ranging from $900 \mathrm{MW}$ to $2459 \mathrm{MW}$. The appropriate numbers of units are committed from the priority loading order for each load condition to meet a probabilistic risk criterion of UCR $<0.001$ [7]. The committed units are loaded using the economic load dispatch method. The CGRR was calculated in 11 decimal places considering all the contingency states and is shown in the third column of Table 3. The table also shows the percent error when the number of contingencies in the evaluation is reduced. It can be seen that the error decreases as the order of the contingencies included in the evaluation is increased. The last column shows that there is no error when CGRR value is expressed 
in 11 decimal places when contingencies up to the fourth order are considered in the evaluation. It can therefore be concluded that considering up to the fourth order contingency in CGRR evaluation provides acceptable results with less than $0.00005 \%$ error.

Table 3. Order of contingency states and accuracy in CGRR evaluation.

\begin{tabular}{ccccccc}
\hline \multirow{2}{*}{ Load (MW) } & \multirow{2}{*}{ No. of Units } & \multirow{2}{*}{ CGRR $\times \mathbf{1 0}^{\mathbf{6}}$} & \multicolumn{4}{c}{ Error (\%) with Included Order of Contingencies } \\
\cline { 4 - 6 } & & & 1st & 2nd & 3rd & 4th \\
\hline 900 & 7 & 2686.33119 & 0.3476 & $3.8 \times 10^{-4}$ & $3.7 \times 10^{-7}$ & 0 \\
1300 & 9 & 2685.91680 & 0.5419 & $1.3 \times 10^{-3}$ & $1.5 \times 10^{-6}$ & 0 \\
1497 & 10 & 2688.12089 & 0.7280 & $1.9 \times 10^{-3}$ & $3.0 \times 10^{-6}$ & 0 \\
1694 & 11 & 2691.40242 & 0.9524 & $3.1 \times 10^{-3}$ & $5.2 \times 10^{-6}$ & 0 \\
2004 & 13 & 2701.18317 & 1.5167 & $6.2 \times 10^{-3}$ & $1.3 \times 10^{-5}$ & 0 \\
2159 & 14 & 2707.68269 & 1.8555 & $8.3 \times 10^{-3}$ & $1.9 \times 10^{-5}$ & 0 \\
2459 & 17 & 2725.82489 & 2.7522 & $1.5 \times 10^{-2}$ & $4.5 \times 10^{-5}$ & 0 \\
\hline
\end{tabular}

\section{Development of a Monte Carlo Simulation Method for CGRR Evaluation}

A sequential Monte Carlo simulation (MCS) method is very useful when the system operation depends on time correlated factors [14]. The times to failure of the committed generating units are assumed to follow an exponential distribution [15]. The initial operating condition is known, and the time to failure $T_{i}$ of each operating unit $i$ is estimated using (8).

$$
T_{i}=-\frac{1}{\lambda_{i}} \ln U_{i}
$$

where $U_{i}$ is a uniformly distributed random number between 0 and 1 .

The MCS method is described with reference to Figure 1, where it is known that at the initial condition of time $t=0$, all the committed $m$ units are in operation. An outage of Unit $i$ occurring after time $T_{i}$ will cause a disturbance within the exposure time $T_{L}$ if $T_{i}<T_{L}$. The response capacity or the regulating margin of the healthy units in the interval $T_{R}$ is calculated to determine if the reduction in loading capacity can be fulfilled by the response capacity. A random number $U_{i}$ is drawn for each committed unit and to simulate the operating performance of the entire operating system. If multiple outages occur within the lead time $T_{L}$, the response capacity of the remaining healthy units within the response time $T_{R}$ following each disturbance is calculated in time chronology, and the simulation run is flagged if the system response capacity fails to fulfill the reduced capacity. The simulations are repeated until convergence, and the numbers of flagged simulation runs are divided by the total number of simulation runs to obtain the CGRR for the operating condition.

\section{Model Validation using Monte Carlo Simulation}

The CGRR results obtained by the proposed analytical technique in Sections 2 and 4 are compared with the results obtained using a sequential Monte Carlo simulation (MCS) method developed in Section 5 in order to validate the proposed method. The analysis is performed on the IEEE-RTS. The priority loading order, failure rate, operating capacity limits, and the operating cost parameters of the IEEE-RTS are given in Table 4 [13]. 
Table 4. IEEE-RTS generating unit data.

\begin{tabular}{cccccccc}
\hline \multirow{2}{*}{$\begin{array}{c}\text { Priority } \\
\text { Order }\end{array}$} & $\begin{array}{c}\text { MCR } \\
\text { (MW) }\end{array}$ & $\begin{array}{c}\text { Min. Capacity } \\
\text { (MW) }\end{array}$ & $\begin{array}{c}\text { Ramp Rate } \\
\text { (MW/min) }\end{array}$ & $\begin{array}{c}\text { Fail. Rate } \\
\text { (occ/yr) }\end{array}$ & \multicolumn{3}{c}{ Cost Parameters (\$/h) } \\
\cline { 7 - 9 } & & & & & $\mathbf{a}$ & $\mathbf{b}$ & $\mathbf{c}$ \\
\hline $1-4$ & 50 & 0 & 10 & 4.42 & 0.000 & 0.500 & 0.00000 \\
$5-6$ & 400 & 200 & 0 & 7.96 & 216.576 & 5.345 & 0.00028 \\
7 & 350 & 150 & 9 & 7.62 & 388.25 & 8.919 & 0.00392 \\
$8-10$ & 197 & 80 & 6 & 9.22 & 301.223 & 20.023 & 0.00300 \\
$11-14$ & 155 & 60 & 5 & 9.13 & 206.703 & 9.271 & 0.00667 \\
$15-17$ & 100 & 40 & 3 & 7.30 & 286.241 & 17.924 & 0.00220 \\
$18-21$ & 76 & 25 & 2 & 4.47 & 100.439 & 12.145 & 0.01131 \\
$22-26$ & 12 & 5 & 1 & 2.98 & 30.396 & 23.278 & 0.13733 \\
$27-30$ & 20 & 6 & 4 & 19.50 & 40.000 & 37.554 & 0.18256 \\
$31-32$ & 50 & 0 & 10 & 4.47 & 0.000 & 0.500 & 0.00000 \\
\hline
\end{tabular}

An operating condition was considered with a load of 1995 MW. Using the conventional N-1 unit commitment criterion, a total of 13 generating units were committed from the priority loading order. An economic load dispatch by gradient method was used to dispatch the committed units. Table 5 shows the unit loadings for the operating condition.

Table 5. A sample operating condition of the IEEE-RTS under study.

\begin{tabular}{cccccccccccccc}
\hline Unit \# & $\mathbf{1}$ & $\mathbf{2}$ & $\mathbf{3}$ & $\mathbf{4}$ & $\mathbf{5}$ & $\mathbf{6}$ & $\mathbf{7}$ & $\mathbf{8}$ & $\mathbf{9}$ & $\mathbf{1 0}$ & $\mathbf{1 1}$ & $\mathbf{1 2}$ & $\mathbf{1 3}$ \\
\hline MCR & 50 & 50 & 50 & 50 & 400 & 400 & 350 & 197 & 197 & 197 & 155 & 155 & 155 \\
Loading & 50 & 50 & 50 & 50 & 400 & 400 & 302 & 80 & 80 & 80 & 151 & 151 & 151 \\
Reserve & 0 & 0 & 0 & 0 & 0 & 0 & 48 & 117 & 117 & 117 & 4 & 4 & 4 \\
RM & 0 & 0 & 0 & 0 & 0 & 0 & 48 & 60 & 60 & 60 & 4 & 4 & 4 \\
\hline \multicolumn{1}{c}{ Note: All data in MW } & & & & & & & &
\end{tabular}

The CGRR results obtained from the two methods are shown in Table 6 in eight decimal places for comparison. It can be seen that the two results are very close. The proposed analytical method is simple to apply and provides very close results to that obtained from a sequential MCS method that simulates the actual operation of a system condition in time chronology.

Table 6. A comparison of the CGRR results of the example IEEE-RTS operating condition.

\begin{tabular}{cc}
\hline Evaluation Method & CGRR \\
\hline Analytical & 0.00270118 \\
MCS & 0.00270114 \\
\hline
\end{tabular}

\section{Impact of Ramp Rate and Failure Rate on CGRR}

The impacts of generating unit ramp rates and the failure rates on the CGRR index provide important information for a power system operator to optimally schedule generating units in system operation. This is investigated by analyzing the IEEE-RTS operating schedule given in Table 5 to serve a load of $1995 \mathrm{MW}$. As mentioned earlier, a total of 13 generating units were committed from priority loading order to meet the $\mathrm{N}-1$ criterion, and the units were loaded based on the economic load dispatch. Four different cases were considered to investigate the impact of ramp rates and failure rates on the CGRR index. The IEEE-RTS data given in Table 4 constitutes the base case, or Case 1 . The failure rates of the committed units are doubled in Case 2. The ramp rates of the committed units are doubled in Case 3, while keeping the failure rates the same as that in the base case. Both the ramp rates and the failure rates are doubled in Case 4. The CGRR was evaluated for the four cases and shown in Figure 2.

Figure 2 shows that CGRR increases significantly as the failure rate of the committed unit is increased. It can be seen that the CGRR increases in similar proportion when the failure rate is doubled 
from case 1 to Case 2. The CGRR increases over four times when the failure rate is doubled from Case 3 to Case 4. Figure 2 shows that the CGRR is highly sensitive to the ramp rate of a committed unit that carries an operating reserve. It can be seen that the CGRR was reduced more than 100 times when the ramp rates of the units were doubled from Case 1 to Case 3 . It should be noted that the ramp rate of the base load generators that do not carry any spinning reserve have no impact on the risk index. This study illustrates that the impact of these parameters should be evaluated prior to making unit commitment and dispatch decisions.

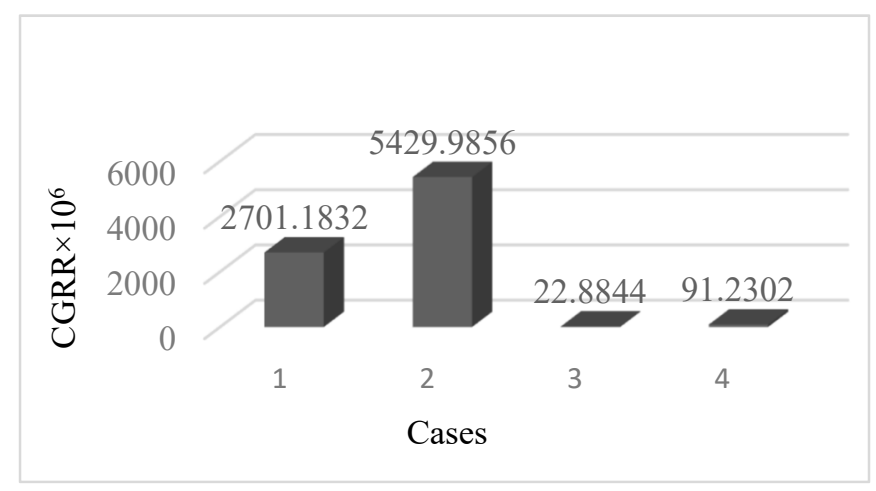

Figure 2. CGRR evaluation considering different ramp rates and failure rates.

\section{Application of CGRR Index in Power System Operating Decisions}

The deterministic N-1 criterion is widely used to assess operating reserve requirement for unit commitment, and the committed units are generally dispatched to share the system load using economic load dispatch. The operating decisions taken based on these deterministic techniques can neither quantify nor provide consistent operating risks for different operating conditions. It will be increasingly important in the future to adopt risk-based methods as uncertainty in power system increases due to ongoing system changes. The presented probabilistic approach and the CGRR index can be used to make risk based operating decisions, as illustrated in this section.

\subsection{CGRR Criterion for Operating Decisions}

It is an important task to determine the acceptable magnitude of operating risk that should be used as a reliability criterion to make operating decisions. In an explicit sense, the operating costs associated with maintaining a specified level of operating reliability should be justified by the worth to the consumers. However, it is not an easy task to determine the reliability worth, and efforts to determine the cost of different magnitudes and duration of interruptions to the various types of consumers have been reported from time to time. Many electric utilities have relied on past performance evaluation to determine the criterion value when adopting a new reliability index. This practical approach is illustrated in this section by considering existing methods for unit commitment and load dispatch to determine an acceptable CGRR criterion for the IEEE-RTS.

The CGRR was evaluated for all the operating conditions of the IEEE-RTS, in which the generating units were committed using the $\mathrm{N}-1$ criterion to meet the entire range of load levels, and the economic load dispatch using the gradient method was used to dispatch the committed units. Figure 3 shows the CGRR of the different operating conditions as the generating units are additionally committed based on their priority loading order to the meet the load as it is increased from its minimum value to its peak.

Figure 3 shows the comprehensive operating risk expressed by the CGRR index generally increases as the committed number of generating units is increased to meet an increase in load. This is due to the increased likelihood of generating unit outages and is influenced by their respective failure rates. The CGRR value also depends on the ramp rates of the committed units carrying spinning reserves, as described earlier. It was, however, found that the effect of unit ramp rate was less significant after a certain load level in the IEEE-RTS as the total regulating margin remained unchanged with further 
addition of newly committed units to meet the increased load. Figure 3 shows that the CGRR varies from 0.002686 to 0.002734 when expressed in six decimal places. An operating condition for an average load of $1900 \mathrm{MW}$ assuming an annual load factor of $67 \%$ results in a CGRR value of 0.002698 , which can be used as the starting CGRR criterion is adopting this new approach in the IEEE-RTS. The criterion can then be revised based on operating experience and customer satisfaction surveys.

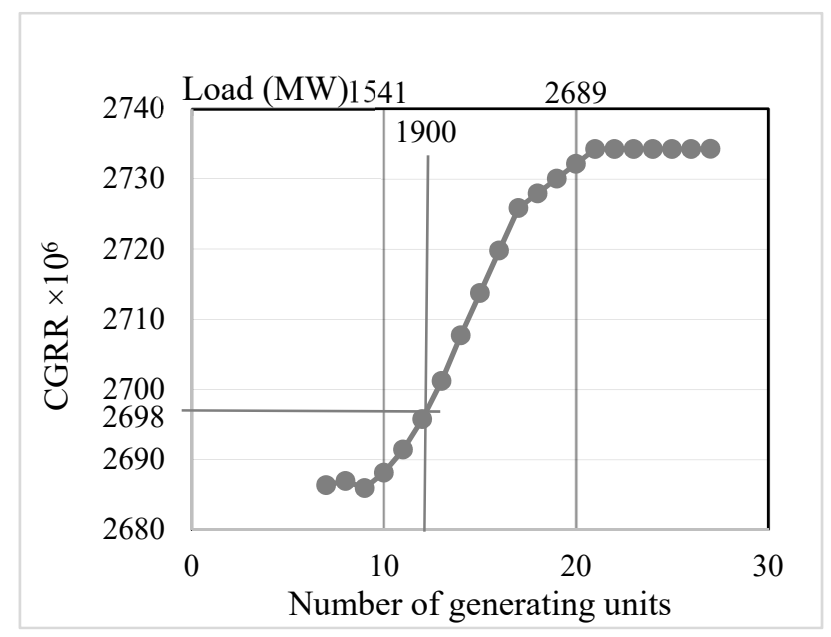

Figure 3. CGRR variation with different operating conditions.

\subsection{System Operation Using CGRR Criterion}

It is recommended that an off-line CGRR evaluation of the past operating conditions of the system be first carried out for a specified period in order to comprehend the operating risks associated with past operator practice and the system operating performance. The off-line assessment can be used to determine the operating conditions with CGRR values significantly above and below the accepted criterion value. For these operating conditions, the load dispatch and/or the unit commitment should be rescheduled and CGRR evaluated until an acceptable value is obtained at the lowest possible cost.

As an example, Figure 3 shows the CGRR of the IEEE-RTS obtained from an off-line evaluation of the different operating conditions as the load is varied from its minimum value to its peak. For example, the operating schedule to satisfy a load of $1995 \mathrm{MW}$ using an N-1 unit commitment and economic load dispatch results in a CGRR of 0.002701 , which exceeds the criterion value of 0.002698 . It is necessary to re-dispatch the units in such a way that the total regulating margin will increase and reduce the CGRR to an acceptable level. It should be noted that the CGRR is also sensitive to the failure rates of the units carrying the regulating margin.

The new load dispatch should be as economical as possible within the risk constraint when the committed units are re-dispatched to meet the CGRR criterion. The potential generating units for rescheduling are grouped into two classes, Class A and B. A Class A unit has a ramping capability greater than its current operating reserve, whereas a Class $B$ unit has an operating reserve greater than its ramping capability. A part of the load from a Class A unit should be transferred to a Class B unit in order to increase the system regulating margin within the response time. This process is carried out in small load steps. The loading from the generator with the highest incremental operating cost in Class A is first transferred to the generator with the lowest incremental operating cost of Class B. After each incremental load transfer, the CGRR is calculated, and the load transfer process is repeated until the desired CGRR value is achieved.

Table 7 illustrates the above described operating condition with 13 generating units committed to meet a load of $1995 \mathrm{MW}$ in the IEEE-RTS. The table shows the economic load dispatch (ELD) and the re-dispatch to meet the CGRR criterion. The Class A and B units are also indicated along with the unit ID numbers in the first column. A part of the load from Class A units 7, 11, 12, and 13 were transferred to Class B units 8, 9, and 10 so that the CGRR criterion was satisfied. The resulting CGRR 
is 0.0026979 which is within the criterion value of 0.002698 . The regulating margins associated with the two dispatches are also shown in the table. It can be seen that an increase in $71 \mathrm{MW}$ of the total regulating margin was required to maintain the acceptable CGRR criterion.

Table 7. Example of economic re-dispatch in the IEEE-RTS operating schedule to meet CGRR criterion.

\begin{tabular}{|c|c|c|c|c|c|c|}
\hline \multirow{2}{*}{ Units } & \multirow{2}{*}{$\begin{array}{l}\text { MCR } \\
\text { (MW) }\end{array}$} & \multirow{2}{*}{$\begin{array}{c}\text { Ramping Capability } \\
\text { (MW) }\end{array}$} & \multicolumn{2}{|c|}{ ELD } & \multicolumn{2}{|c|}{ Re-Dispatch } \\
\hline & & & Loading (MW) & RM (MW) & Loading (MW) & RM (MW) \\
\hline $1-\mathrm{A}$ & 50 & 50 & 50 & 0 & 50 & 0 \\
\hline $2-\mathrm{A}$ & 50 & 50 & 50 & 0 & 50 & 0 \\
\hline 3-A & 50 & 50 & 50 & 0 & 50 & 0 \\
\hline $4-\mathrm{A}$ & 50 & 50 & 50 & 0 & 50 & 0 \\
\hline 5 & 400 & 0 & 400 & 0 & 400 & 0 \\
\hline 6 & 400 & 0 & 400 & 0 & 400 & 0 \\
\hline $7-\mathrm{A}$ & 350 & 90 & 302 & 48 & 276 & 74 \\
\hline $8-B$ & 197 & 60 & 80 & 60 & 104 & 60 \\
\hline $9-B$ & 197 & 60 & 80 & 60 & 104 & 60 \\
\hline $10-B$ & 197 & 60 & 80 & 60 & 104 & 60 \\
\hline 11-A & 155 & 50 & 151 & 4 & 135 & 19 \\
\hline $12-\mathrm{A}$ & 155 & 50 & 151 & 4 & 136 & 19 \\
\hline $13-\mathrm{A}$ & 155 & 50 & 151 & 4 & 136 & 19 \\
\hline Total & 2406 & 620 & 1995 & 240 & 1995 & 311 \\
\hline
\end{tabular}

The off-line evaluation and results provide useful information to understand the required changes in unit commitment and load dispatch in order to maintain the CGRR at an acceptable level as operating conditions change over time. This creates a knowledge base to implement the CGRR approach in real time, applying predictive evaluation using the proposed techniques.

The decision to make the transition to the new probabilistic approach in a continuously operating power system can begin at an operating condition, at which the CGRR value is within the acceptable level. The presented method can then be applied in real-time with repeated calculation of the CGRR by incorporating short-term forecast of system changes. This process is illustrated by the algorithm in Figure 4.

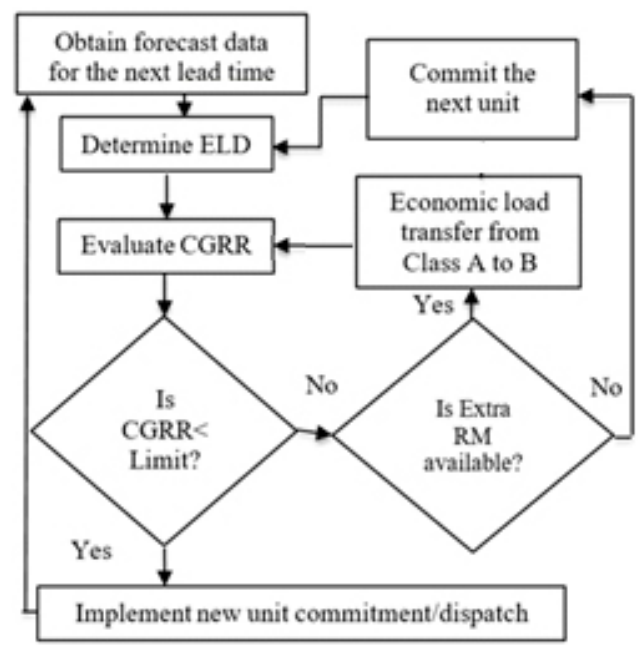

Figure 4. Algorithm for implementing CGRR criterion in operating decisions.

The next operating decision in an operating condition is initiated by the short-term forecast of system changes within the lead time of the ready to start generating units available in the system. The change in loading to satisfy the system change is first determined using an economic load dispatch. The CGRR for the new economic load dispatch is evaluated and compared with the accepted criterion, as shown in 
Figure 4. This new dispatch action is taken if the CGRR criterion is satisfied. If the criterion is not met, the economic dispatch is modified by transferring the load from the class A units to the class B units one step at a time in a recursive approach until the CGRR criterion is satisfied. A decision is then taken to implement the resulting load dispatch that meets the risk criterion. If the system does not have adequate regulate the margin to meet the CGRR criterion, the commitment of the next available standby unit with the lowest incremental operating cost is considered as the additional generating unit in the CGRR evaluation, and the load dispatch that results in an acceptable CGRR value is determined by repeating the above described process, as shown in the algorithm. A decision is then taken to commit the selected additional unit. The CGRR evaluation and the implementation decision proceed continuously in time chronology in order to consistently maintain the CGRR within the acceptable criterion value.

The algorithm in Figure 4 is further illustrated with an example of an IEEE-RTS operating condition, in which 13 generating units are committed from the priority loading order. The total committed generation capacity is $2406 \mathrm{MW}$. In this example, the failure rates of the individual generating units are assumed to be 1.5 times that of their original values. It is assumed that the short-term system load forecast is $2006 \mathrm{MW}$ in this example. The economic load dispatch (ELD) of the committed 13 units to meet the forecast load is shown in the fourth column of Table 8. The total regulating margin is $230 \mathrm{MW}$, and the resulting CGRR is 0.004062 . These results are shown in the first row of Table 9. The operating risk is unacceptable as it is well above the criterion value of 0.002698 . Following the algorithm in Figure 4 , a re-dispatch is considered, since the total regulating margin can be increased by economic load transfer from the class A units 11, 12, and 13 to class B units 7, 8, 9, and 10. The economic re-patch to lower the operating risk is shown in the sixth column of Table 8. The CGRR result and the total regulating margin is shown in the second row of Table 9. The new CGRR is 0.002740, which is also unacceptable. The regulating margin is increased by $168 \mathrm{MW}$ and cannot be further increased due to loading constraints of the committed units. In order to further decrease the CGRR, the commitment of an additional generating unit is considered, as shown in the algorithm of Figure 4 . The eighth column in Table 8 shows the economic load dispatch (ELD) of the committed units, considering the addition of a $155 \mathrm{MW}$ unit that has a failure rate of 9.13 occurrences per year. The total regulating margin is increased to $355 \mathrm{MW}$, and the resulting CGRR is 0.002742 , as shown in the third row of Table 9. A re-dispatch is again considered since the CGRR still exceeds the criterion value. An economic load transfer evaluation is carried out by transferring part of the load from the class A units $11,12,13$, and 14 to class B units 8,9 , and 10. The economic re-dispatch of the 14 generating units to lower the operating risk is shown in the tenth column of Table 8 . The new CGRR is 0.000058 , which is within the acceptable criterion.

Table 8. IEEE-RTS system of 2006 MW load with different operating conditions.

\begin{tabular}{ccccccccccc}
\hline \multirow{2}{*}{ Unit } & \multirow{2}{*}{ MCR } & \multirow{2}{*}{$\begin{array}{c}\text { Ramping } \\
\text { Capability }\end{array}$} & \multicolumn{2}{c}{ 13 Units ELD } & \multicolumn{2}{c}{$\begin{array}{c}\text { 13 Units } \\
\text { Re-Dispatch }\end{array}$} & 14 Units ELD & \multicolumn{2}{c}{$\begin{array}{c}\text { 14 Units } \\
\text { Re-Dispatch }\end{array}$} \\
\cline { 4 - 11 } & & & Loading & RM & Loading & RM & Loading & RM & Loading & RM \\
\hline 1 & 50 & 50 & 50 & 0 & 50 & 0 & 50 & 0 & 50 & 0 \\
2 & 50 & 50 & 50 & 0 & 50 & 0 & 50 & 0 & 50 & 0 \\
3 & 50 & 50 & 50 & 0 & 50 & 0 & 50 & 0 & 50 & 0 \\
4 & 50 & 50 & 50 & 0 & 50 & 0 & 50 & 0 & 50 & 0 \\
5 & 400 & 0 & 400 & 0 & 400 & 0 & 400 & 0 & 400 & 0 \\
6 & 400 & 0 & 400 & 0 & 400 & 0 & 400 & 0 & 400 & 0 \\
7 & 350 & 90 & 306 & 44 & 261 & 89 & 260 & 90 & 260 & 90 \\
8 & 197 & 60 & 80 & 60 & 136 & 60 & 80 & 60 & 86 & 60 \\
9 & 197 & 60 & 80 & 60 & 136 & 60 & 80 & 60 & 86 & 60 \\
10 & 197 & 60 & 80 & 60 & 136 & 60 & 80 & 60 & 86 & 60 \\
11 & 155 & 50 & 153 & 2 & 112 & 43 & 126 & 29 & 121 & 34 \\
12 & 155 & 50 & 153 & 2 & 112 & 43 & 126 & 29 & 121 & 33 \\
13 & 155 & 50 & 153 & 2 & 112 & 43 & 126 & 29 & 122 & 33 \\
14 & 155 & 50 & & & & & 126 & 29 & 122 & 33 \\
\hline
\end{tabular}


Table 9 summarizes the CGRR results obtained during the evaluation process using the recursive algorithm of Figure 4. The total regulating margin and the operating costs associated with the different schedules considered during the evaluation process is also shown in the table. The study results show that the dispatch of 13 generating units that are committed using the $\mathrm{N}-1$ criterion to meet the load cannot satisfy the CGRR criterion. The CGRR criterion can only be satisfied by committing an additional generating unit with an increased cost, as shown in Table 9. The table also shows the increase in operating cost as the dispatch is changed from ELD in order to decrease the operating risk. The first row represents the operating decision that would be taken using the existing deterministic methods, which results in an operating cost of $19,505 \$ / \mathrm{h}$. The operating risk is however very high. The fourth row in Table 9 represents the operating decision taken using the proposed probabilistic methods, which results in a low operating risk that satisfies the CGRR criterion. The operating cost is however increased by $250 \$ / \mathrm{h}$. Table 9 also shows the CGRR and cost results when two additional units are committed. It can be seen that this operating decision is not justified by the high operating cost. The results from this study show that an increase in operating reliability or a decrease in CGRR can be achieved with an increase in operating costs. A useful contribution of the proposed approach is that it can quantify the operating reliability benefit that can be compared with the operating cost, and therefore provide useful indicators to value-based operating decisions.

Table 9. Example results for operating reliability and cost comparison.

\begin{tabular}{cccc}
\hline Committed Units and Dispatch & Total RM (MW) & CGRR $\times \mathbf{1 0}^{\mathbf{6}}$ & Cost (\$/h) \\
\hline 13 units, ELD & 230 & 4062.161706 & $19,505.11$ \\
13 units, re-dispatch & 396 & 2740.451292 & $21,118.19$ \\
14 units, ELD & 355 & 2742.428945 & $19,582.40$ \\
14 units re-dispatch & 401 & 57.65131996 & $19,755.09$ \\
15 units ELD & 442 & 60.95279158 & $20,150.68$ \\
\hline
\end{tabular}

\section{Conclusions}

The reliability of power supply to consumers largely depends on operating decisions taken to withstand anticipated and random generation and load fluctuations. The concerns with growing uncertainty in power system conditions can be addressed using a probabilistic approach that can quantify the degree of operating risk and be used in decision making. The CGRR index proposed in this paper is responsive to the uncertainty of varying magnitudes of power disturbances, the amount of operating reserve in the system, ramp rates and responding capacity of the units carrying the reserve, and the lead time, after which the system will receive further capacity assistance. The index therefore provides a comprehensive risk assessment that should be useful in operating decisions with unit commitment and load dispatch. The paper presents an analytical method to evaluate the index, and an application of the method is illustrated on a test system. The paper also presents a Monte Carlo simulation method for CGRR evaluation which was then used to validate the analytical method. A suitable CGRR criterion can be evaluated based on past acceptable performance, as illustrated in the paper. The criterion can then be used to operate the system at a consistent level of risk. The results show that limiting the number of contingencies to the fourth order in the analytical method can significantly reduce the computation burden without sacrificing reasonable accuracy, and therefore, be used in real-time CGRR evaluation during system operation. Offline evaluation results from all potential operating conditions will provide valuable inputs to real-time evaluation and decisions. An increase in operating reliability often requires additional operating costs. The proposed methodology and index can quantify the operating reliability benefit and be compared with the operating cost to provide useful indicators to value-based operating decisions.

Author Contributions: The research work leading to the paper was carried out by N.N. with close supervision from R.K. 
Funding: The authors acknowledge the financial support from the Natural Sciences and Engineering Research Council of Canada in funding this research.

Conflicts of Interest: The authors declare no conflict of interest.

\section{References}

1. Billinton, R.; Allan, R.N. Reliability Evaluation of Power Systems, 2nd ed.; Plenum: New York, NY, USA, 1996.

2. North American Electric Reliability Corporation (NERC). Balancing and Frequency Control; A Technical Document Prepared by the NERC Resources Subcommittee; NERC: Princeton, NJ, USA, 2011.

3. Anstine, L.T.; Burke, R.E.; Casey, J.E.; Holgate, R.; John, R.S.; Stewart, H.G. Application of Probability Methods to the Determination of Spinning Reserve Requirements for the Pennsylvania-New Jersey-Maryland Interconnection. IEEE Trans. Power Appar. Syst. 1963, 82, 726-735. [CrossRef]

4. Billinton, R.; Jain, A.V. The Effect of Rapid Start and Hot Reserve Units in Spinning Reserve Studies. IEEE Trans. Power Appar. Syst. 1972, PAS-91, 511-516. [CrossRef]

5. Chowdhury, N.A.; Billinton, R. Unit Commitment in Interconnected Generating Systems Using a Probabilistic Technique. IEEE Trans. Power Appar. Syst 1990, 5, 1231-1238. [CrossRef]

6. Khan, M.E.; Billinton, R. Composite System Spinning Reserve Assessment in Interconnected Systems. IET Proc.-Gener. Transm. Distrib. 1995, 142, 305-309. [CrossRef]

7. Billinton, R.; Fotuhi-Firuzabad, M. A Basic Framework for Generating System Operating Health Analysis. IEEE Trans. Power Syst. 1994, 9, 1610-1617. [CrossRef]

8. Billinton, R.; Karki, B.; Karki, R.; Ramakrishna, G. Unit Commitment Risk Analysis of Wind Integrated Power Systems. IEEE Trans. Power Syst. 2009, 24, 930-939. [CrossRef]

9. Jaefari-Nokandi, M.; Monsef, H. Scheduling of Spinning Reserve Considering Customer Choice on Reliability. IEEE Trans. Power Syst. 2009, 24, 1780-1789. [CrossRef]

10. Jain, A.V.; Billinton, R. Spinning Reserve Allocation in a Complex Power System. In Proceedings of the IEEE Winter Power Meeting, New York, NY, USA, 28 January-2 February 1973; pp. 1-8.

11. Yang, M.; Han, X.; Jiang, Z.; Cheng, Y.; Lee, W. A Novel Method for Advanced Dispatch Considering Response Risk Constraints. In Proceedings of the North American Power Symposium (NAPS), Starkville, MS, USA, 4-6 October 2009.

12. Abiri-Jahromi, A.; Fotuhi-Firuzabad, M. Optimal Scheduling of Spinning Reserve Based on Well-Being Model. IEEE Trans. Power Syst. 2007, 22, 2048-2057. [CrossRef]

13. Subcommittee, P.M. IEEE Reliability Test System. IEEE Trans. Power Appar. Syst. 1979, PAS-98, $2047-2054$. [CrossRef]

14. Billinton, R.; Jonnavithula, A. Application of Sequential Monte Carlo Simulation to Evaluation of Distributions of Composite System Indices. IET Proc.-Gener. Transm. Distrib. 1997, 144, 87-90. [CrossRef]

15. Billinton, R.; Li, W. Reliability Assessment of Electrical Power System Using Monte Carlo Methods; Plenum Press: New York, NY, USA, 1994.

(C) 2019 by the authors. Licensee MDPI, Basel, Switzerland. This article is an open access article distributed under the terms and conditions of the Creative Commons Attribution (CC BY) license (http://creativecommons.org/licenses/by/4.0/). 